\title{
Enhanced MDIR Receiver for Space-Time Dispersive Channels
}

\author{
Josep Vidal, Member, IEEE, Margarita Cabrera, Adrian Agustín de Dios, and Miguel Angel Lagunas, Fellow, IEEE
}

\begin{abstract}
A particular property of the matched desired impulse response receiver is introduced in this paper, namely, the fact that full exploitation of the diversity is obtained with multiple beamformers when the channel is spatially and timely dispersive. This particularity makes the receiver specially suitable for mobile and underwater communications. The new structure provides better performance than conventional and weighted VRAKE receivers, and a diversity gain with no needs of additional radio frequency equipment. The baseband hardware needed for this new receiver may be obtained through reconfigurability of the RAKE architectures available at the base station. The proposed receiver is tested through simulations assuming UTRA frequency-division-duplexing mode.
\end{abstract}

Index Terms-Adaptive antennas, multipath channels, multiuser/single-user receivers, wireless communications.

\section{INTRODUCTION}

$\mathbf{T}$ HE ADVENT of the third-generation of mobile communications systems has been accompanied by the recognition of the large increase in system capacity that can be obtained from the use of adaptive antenna arrays. Care has been taken in the definition of the standard to include capabilities for space-time processing of the uplink and downlink. Therefore, a panoply of receivers have arisen [1], [5], [6], [8] which improved the performance of the single sensor receiver without conveying the complexity of the optimum multiuser receiver [12]. One of the most celebrated receivers in this trading is the matched desired impulse response (MDIR) [6] whose capabilities for reconfigurability and use in both time-division-duplexing (TDD) and frequency-division-duplexing (FDD) modes have been demonstrated [7]. Some unpublished properties of this receiver are reported here, which allow further exploitation of the diversity present in a spatio-temporal dispersive channel.

Section II defines the signal model for the uplink channel. Section III shows two possibilities for spatial front-ends: a noise-plus-interference matrix inversion (NIMI) receiver, which can be considered as a reduced complexity weighted VRAKE receiver and the proposed extended MDIR (EMDIR).

Manuscript received February 23, 2001; revised December 21, 2001; accepted February 26, 2002. The editor coordinating the review of this paper and approving it for publication is L. Hanzo. This work was supported in part by the Spanish Government under Grant TIC98-0703, Grant TIC99-0849, Grant TIC2000-1025, and Grant FIT-070000-2000-649, in part by the Generalitat of Catalunya under Grant 2000SGR 00083, and in part by the AECI (Programa de Cooperación Interuniversitaria Hispano-Marroquí).

The authors are with the Department of Signal Theory and Communications, Universitat Politècnica de Catalunya, 08034 Barcelona, Spain (e-mail pepe@gps.tsc.upc.es; marga@gps.tsc.upc.es; agustin@gps.tsc.upc.es).

Digital Object Identifier 10.1109/TWC.2002.806369
Similarities and differences between them are shown and performance compared in terms of bit-error rate (BER) equations for the Gaussian channel are given in Section IV. Simulations in Section $\mathrm{V}$ will demonstrate the superiority of the EMDIR receiver in realistic conditions. Results show a significant improvement in the probability of error with respect to conventional approaches, that is, only spatial beamforming or only VRAKE combining. Section VI concludes the paper.

\section{SignAl MODEL}

The single-user signal model assumed for the signal received at $M$ sensors after matched filtering and chip-time sampling may be written in column vector form as

$$
\mathbf{y}=\overline{\mathbf{H}} \mathbf{d}+\mathbf{w} \text {. }
$$

Assuming the following definitions:

- channel length is $L$ chips;

- $N_{t}$ and $N_{p}$ are the number of traffic and pilot symbols, respectively;

- $N$ is the number of chips per slot;

- $Q_{p}$ and $Q_{t}$ are the spreading factor of the pilot and traffic channels, respectively,

then, each term is defined as (2), shown at the bottom of the next page. $\overline{\mathbf{H}}$ is the space-time channel of the desired user whose size is $M\left(N_{p} Q_{p}+N_{t} Q_{t}+2 L-2\right) \times\left(N_{p}+N_{t}\right), \mathbf{d}$ [of size $\left(N_{p}+N_{t}\right) \times 1$ ] includes the traffic symbols $\left(\mathbf{d}_{t}\right)$ and the pilot symbols $\left(\mathbf{d}_{p}\right)$ (in case they are I/Q multiplexed) for this user and $\mathbf{w}$ is the vector accounting for noise plus interferences, of size $((N M) \times 1)$. Matrix $\overline{\mathbf{H}}_{i}$ contains the convolution of the impulse response of the propagation channel seen by sensor $i$ (computed at chip time) and the spreading code. The effect of the long scrambling code can be represented by the time variation of the spreading code from symbol to symbol, which is denoted with the superscript $(k)$

$$
\begin{aligned}
& h_{i, p}^{(k)}(n)=h_{i}(n) * c_{p}^{(k)}(n) \\
& h_{i, t}^{(k)}(n)=h_{i}(n) * c_{t}^{(k)}(n) .
\end{aligned}
$$

In (2), five symbols have been plotted for the pilot channel and ten for the traffic channel.

\section{SPATIAL FRONT-ENDS}

\section{A. NIMI Receiver}

With this model in mind and assuming the interference-plusnoise as spatially and temporally correlated Gaussian noise, it 
is possible to formulate the likelihood function which, appropriately minimized, gives the detector for the unknown symbols

$$
\begin{aligned}
J & =(\mathbf{y}-\overline{\mathbf{H}} \mathbf{d})^{H} \mathbf{R}_{w}^{-1}(\mathbf{y}-\overline{\mathbf{H}} \mathbf{d}) \\
& =\mathbf{y}^{H} \mathbf{R}_{w}^{-1} \mathbf{y}-2 \operatorname{Re}\left\{\mathbf{y}^{H} \mathbf{R}_{w}^{-1} \overline{\mathbf{H}} \mathbf{d}\right\}+\mathbf{d}^{H} \overline{\mathbf{H}}^{H} \mathbf{R}_{w}^{-1} \overline{\mathbf{H}} \mathbf{d}
\end{aligned}
$$

where $\mathbf{R}_{w}$ is the correlation matrix of the space-time noise vector $\mathbf{w}$. Some assumptions are possible so as to simplify the receiver.

A.1) The correlation matrix of the noise-plus-interference could be decomposed as the Kronecker product [2] of a spatial correlation matrix and a temporal correlation matrix

$$
\mathbf{R}_{w}=\mathbf{R}_{w, s} \otimes \mathbf{R}_{w, t}
$$

which agrees with recent channel models [9], in which the time and angular spreads are shown to be independent phenomena.

A.2) The physical channel length ( $L$ chips) is much shorter than the length of the spreading code (which is the case when designing a direct-sequence code-division multiple-access (DSCDMA) system), so the matrix $\overline{\mathbf{H}}^{H} \mathbf{R}_{w}^{-1} \overline{\mathbf{H}}$ is almost diagonal and the last term in (4) can be neglected in its minimization. In fact, this is one of the reasons why high bit-rate users allocated in highly dispersive rural or hilly environments (where delay spreads are usually long compared to the length of the spreading codes) suffer from very poor performance.

As in the optimization of (4) with respect to d the first term can be discarded, only the middle term remains in (4) and it constitutes a sufficient statistics of the problem. Its maximization leads to the well-known Rake receiver when both space and time correlation matrices are assumed white

$$
\begin{aligned}
\hat{\mathbf{d}} & =\arg \max \operatorname{Re}\{\Im\} \\
\Im & =\mathbf{y}^{H} \mathbf{R}_{w}^{-1} \overline{\mathbf{H}} \mathbf{d}=\mathbf{y}^{H}\left(\mathbf{R}_{w, s}^{-1} \otimes \mathbf{R}_{w, t}^{-1}\right) \overline{\mathbf{H}} \mathbf{d} \\
& =\mathbf{y}^{H}\left(\mathbf{R}_{w, s}^{-1 / 2} \otimes \mathbf{R}_{w, t}^{-1 / 2}\right)\left(\mathbf{R}_{w, s}^{-1 / 2} \otimes \mathbf{R}_{w, t}^{-1 / 2}\right)^{H} \overline{\mathbf{H}} \mathbf{d} \\
& =\mathbf{y}_{B}^{H} \overline{\mathbf{H}}_{B} \mathbf{d} .
\end{aligned}
$$

The introduction of the correlation matrix of $\mathbf{w}$ implies a prewhitening of both the signal vector $\mathbf{y}$ (which is noted with $\mathbf{y}_{B}$ ) and the desired user channel matrix $\overline{\mathbf{H}}$ (which is noted with $\left.\overline{\mathbf{H}}_{B}\right)$. This operation can be done separately in time and space (note that $\mathbf{R}_{w, s}^{-1 / 2}$ apply only on the spatial components of $\mathbf{y}$ and $\mathbf{R}_{w, t}^{-1 / 2}$ apply on the temporal components).

Of course, this receiver could be fully implemented by using sample estimates of both correlation matrices, but it is usually the case that the complexity of the resulting structures does not justify the improvement obtained with respect to simplified versions. These different receivers can be formulated from (6) by doing certain approximations on the correlation matrices.

A.3) Temporally white interference. It is a reasonable assumption if the number of interfering users is high.

A.4) In general, it cannot be considered that the interference be spatially white. This assumption is realistic only in the case of a very high number of interferers or in a highly angular dispersive scenario. Then, the receiver becomes the optimum vector receiver [11] which will be called VRAKE in the sequel. Otherwise, the matrix $\mathbf{R}_{w, s}$ has to be taken into account, yielding a weighted VRAKE receiver. If temporal whiteness for the interference is assumed (and after some Kronecker algebra [2]), the overall receiver operation can be written as

$$
\Im=\sum_{i=1}^{R} \frac{1}{\sigma_{i}} \mathbf{y}^{H}\left(\mathbf{b}_{i}^{*} \otimes \mathbf{I}\right)\left(\mathbf{b}_{i}^{T} \otimes \mathbf{I}\right) \overline{\mathbf{H d}} .
$$

It is illustrative to interpret (7) as a coherent combining (maximum ratio combining) of the outputs of $R$ beamformers (see Fig. 1), where the eigenvalue $\sigma_{i}$ associated to the beamformer $i$ gives a measure of the reliability of the information conveyed by the branch $i$ of the combiner. The nature of this receiver is easily seen from a simple case: assume the case of $P<M$ point interferers. If vectors $\mathbf{b}_{i}$ are taken as the noise eigenvectors of $\mathbf{R}_{w, s}$ each one acts as a spatial interference canceler.

If for some branches the term $\sigma$ is too high (corresponding to a branch with high noise level), the summation in (7) can be truncated, an operation which is equivalent to a reduced rank approximation of $\mathbf{R}_{w, s}$ [3]

$$
\mathbf{R}_{w, s}^{-1} \cong \mathbf{B} \Sigma^{-1} \mathbf{B}^{H}, \quad \text { where } \mathbf{B} \notin \mathbb{C}^{M \times R} \text { with } R<M
$$

where matrix $\mathbf{B}$ contains the eigenvectors of $\mathbf{R}_{w, s}$ in its columns, and the diagonal matrix $\boldsymbol{\Sigma}$ contains the eigenvalues $\sigma_{i}$. Therefore, the NIMI receiver is nothing more than a reduced rank approximation of a weighted VRAKE.

$$
\begin{aligned}
& \mathbf{d}^{T}=\left[\begin{array}{lll}
\mathbf{d}_{p}^{T} \mathbf{d}_{t}^{T}
\end{array}\right] \\
& \mathbf{y}^{T}=\left[\begin{array}{llll}
\mathbf{y}_{1}^{T} & \mathbf{y}_{2}^{T} & \cdots & \mathbf{y}_{M}^{T}
\end{array}\right] \\
& \mathbf{y}_{i}^{T}=\left[\begin{array}{llll}
y_{i}(0) & y_{i}(1) & \cdots & y_{i}(N-1)
\end{array}\right] \\
& \mathbf{H}=\left[\begin{array}{c}
\overline{\mathbf{H}}_{1} \\
\mathbf{H}_{2} \\
\hdashline \vdots \\
\mathbf{H}_{M}
\end{array}\right]
\end{aligned}
$$

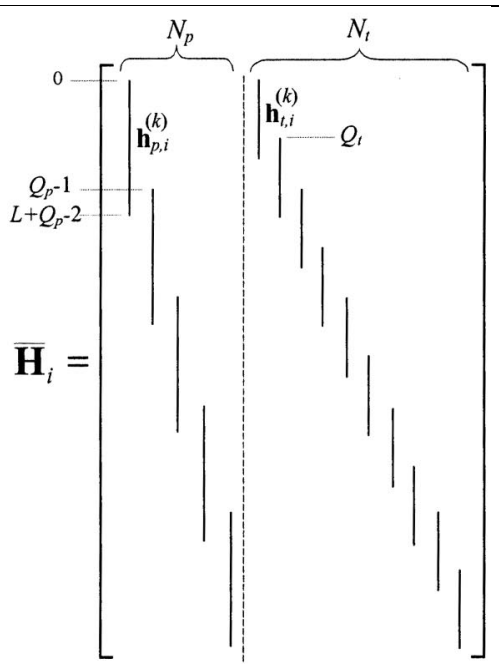




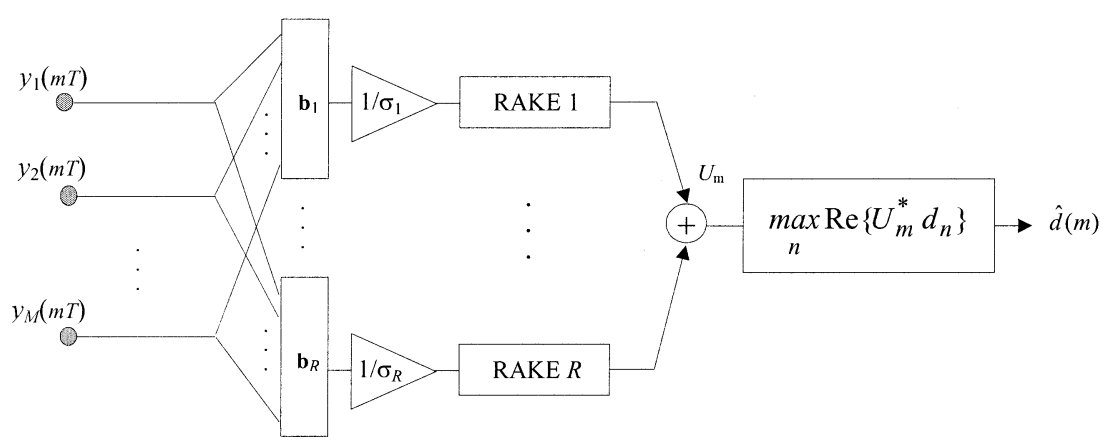

Fig. 1. Optimum combining receiver with reduced rank approximation $(\mathbf{M}>\mathbf{R})$. The gains at each branch are given by the eigenvalues of the noise-plus-interference spatial correlation matrix for the NIMI receiver.

Knowledge of the matrix $\mathbf{R}_{w, s}$ has been assumed which is not a trivial problem. Considerations on its estimation for the FDD mode of UMTS are found in Appendix I.

\section{B. EMDIR Receiver}

A different way to build a spatial front-end is to obtain a spatial combiner $\mathbf{b}$ that maximizes the signal-to-interference-plus noise ratio (SINR) at its output. Let us first redefine the signal model equation as

$$
\mathbf{Y}=\operatorname{unvec}_{(N-L+1) \times M}(\mathbf{y})=\mathbf{D H}+\mathbf{W}
$$

where the operator $\operatorname{unvec}_{a \times b}($.$) rearranges a vector of a b$ elements into a matrix of $a \times b$ elements column-wise. Taking this into account, it is possible to rewrite (1) as in (9), where matrix D is a Toeplitz matrix built at chip time from QPSK complex spread and scrambled symbols

$$
\begin{aligned}
\mathbf{D} & =\left[\begin{array}{cccc}
d(L-1) & d(L-2) & \cdots & d(0) \\
d(L) & d(L-1) & \cdots & d(1) \\
\vdots & \vdots & \ddots & \vdots \\
d(N-1) & d(N-2) & \cdots & d(N-L-1)
\end{array}\right] \\
& \in \mathbb{C}^{(N-L+1) \times L}
\end{aligned}
$$

where $N$ and $L$ where previously defined, and all terms $d(n)$ belong to the set $\{-1-j,-1+j, 1+j, 1-j\}$. The columns of matrix $\mathbf{W}$ contain the noise samples measured at each sensor.

It is usually the case that the symbols (chip level symbols, scrambled with long codes in the FDD mode of UTRA) in matrix $\mathbf{D}$ are uncorrelated, so

$$
\mathbf{D}^{H} \mathbf{D} \cong K \mathbf{I}
$$

where $K$ is $N-L+1$. H contains (column-wise) the $L$-length response of the physical propagation channel seen at each sensor

$$
\begin{aligned}
\mathbf{H} & =\left[\begin{array}{llll}
\mathbf{h}_{1} & \mathbf{h}_{2} & \cdots & \mathbf{h}_{M}
\end{array}\right] \in \mathbb{C}^{L \times M} \\
\mathbf{h}_{i}^{T} & =\left[\begin{array}{llll}
h_{i}(0) & h_{i}(1) & \cdots & h_{i}(L-1)
\end{array}\right] .
\end{aligned}
$$

The MDIR receiver developed in [6] is then given by

$$
\min _{\mathbf{b}} \mathbf{b}^{H} \mathbf{R}_{w, s} \mathbf{b} \quad \text { subject to } \mathbf{b}^{H} \mathbf{H}^{H} \mathbf{D}^{H} \mathbf{D H b}=K \text {. }
$$

It may be shown [6] that the choice of the beamformer is obtained as the generalized eigenvector $\mathbf{b}$ of

$$
\mathbf{R}_{w, s} \mathbf{b}=\lambda \mathbf{H}^{H} \mathbf{D}^{H} \mathbf{D H} \mathbf{b}
$$

associated to the minimum eigenvalue $\lambda$, and the impulse response seen at the output of the beamformer $\mathbf{b}_{j}$ is $\tilde{\mathbf{h}}_{j}=\mathbf{H} \mathbf{b}_{j}$. Note that these $\mathbf{b}$ vectors are different to those in (7). Being non null the signal power at the output of the beamformer $\mathbf{b}_{j}$ through the restriction in (13), $\lambda_{j}$ takes the value of the inverse of the signal-plus-noise-plus-interference power at the output of the associated beamformer [6]. Since the solution provides $M$ beamformers (each giving different SINR at its output), a straightforward question arises: could the different beamformer outputs be efficiently combined as in Fig. 1? Or, in other words, is there any diversity inherent to the multiple beamformers obtained from the MDIR solution? The answer is positive and is given by the following property.

Property 1: The noises at the output of the $M$ beamformers obtained from (14) are uncorrelated, unless the eigenvalues associated are equal.

Proof: Let us extend (14) with all the eigenvectors (contained in matrix B) as

$$
\mathbf{R}_{w, s} \mathbf{B}=\mathbf{H}^{H} \mathbf{D}^{H} \mathbf{D H B} \boldsymbol{\Lambda}=\mathbf{R}_{d, s} \mathbf{B} \boldsymbol{\Lambda}
$$

where $\Lambda$ contains the eigenvalues $\lambda$ of (14). By left-multiplying with the conjugate transpose of $\mathbf{B}$, we obtain on the left hand side of the equation, the correlation matrix of the noises at the output of the different beamformers

$$
\mathbf{B}^{H} \mathbf{R}_{w, s} \mathbf{B}=\mathbf{B}^{H} \mathbf{R}_{d, s} \mathbf{B} \boldsymbol{\Lambda}=\mathbf{\Lambda} \mathbf{B}^{H} \mathbf{R}_{d, s} \mathbf{B} .
$$

We have used in the last equality the fact that the left-hand side of the equation is an Hermitian matrix and the eigenvalues are real. With no loss of generality, let us assume that $\boldsymbol{\Lambda}$ has one multiple eigenvalue $\lambda$, so since the product above is commutative it can be written as

$$
\begin{aligned}
& \boldsymbol{\Lambda} \mathbf{B}^{H} \mathbf{R}_{d, s} \mathbf{B} \\
& =\left[\begin{array}{cc}
\lambda \mathbf{I} & \mathbf{0} \\
\mathbf{0} & \boldsymbol{\Lambda}^{\prime}
\end{array}\right]\left[\begin{array}{cc}
\mathbf{C} & \mathbf{D}^{H} \\
\mathbf{D} & \mathbf{E}
\end{array}\right]=\left[\begin{array}{cc}
\mathbf{C} & \mathbf{D} \\
\mathbf{D}^{H} & \mathbf{E}
\end{array}\right]\left[\begin{array}{cc}
\lambda \mathbf{I} & \mathbf{0} \\
\mathbf{0} & \mathbf{\Lambda}^{\prime}
\end{array}\right] .
\end{aligned}
$$

By explicitly rewriting the matrix product it is easy to see that $\mathbf{D}=\mathbf{0}$ and that $\mathbf{E}$ has to be diagonal. Therefore, we can conclude that

$$
\mathbf{B}^{H} \mathbf{R}_{w, s} \mathbf{B}=\left[\begin{array}{cc}
\lambda \mathbf{C} & \mathbf{0} \\
\mathbf{0} & \boldsymbol{\Lambda}^{\prime \prime}
\end{array}\right] \quad \text { where } \boldsymbol{\Lambda}^{\prime \prime} \text { is diagonal. }
$$

Therefore, we can use the multiple beamformers provided by the MDIR receiver and coherently combine their outputs according to Fig. 1, as long as the associated eigenvalues are not equal. Note however, that the number of eigenvectors given by (14) 
depend on the rank of the signal matrix $\mathbf{H}^{H} \mathbf{D}^{H} \mathbf{D H}$ (assuming full rank of the noise-plus-interference matrix). A rank higher than one, implies the existence of both spatial and temporal dispersion in the desired user.

Another property of interest for the analysis of the performance of the MDIR receiver is the following.

Property 2: The channels associated to the beamformers having different eigenvalues $\tilde{\mathbf{h}}_{i}=\mathbf{H b}_{i}$ are orthonormal, that is, $\tilde{\mathbf{h}}_{i}^{H} \tilde{\mathbf{h}}_{j}=\delta_{i-j}$, provided that the transmitted symbols are uncorrelated [see (11)]. $\delta_{i}$ is the Kronecker delta.

Proof: The diagonality of matrix $\mathbf{B}^{H} \mathbf{H}^{H} \mathbf{D}^{H} \mathbf{D H B}$ stated in Property 1 (17), along with the restriction in (13) completes the proof.

\section{BER EQUATIONS}

BER equations are derived here for the two receivers, assuming Gaussian temporally white interference. This assumption is quite restrictive and conclusions should be drawn carefully, but it allows the comparison of both receivers analytically.

\section{A. NIMI Receiver}

For the $M$ sensor NIMI receiver, the multiple beamformer operation in Fig. 1 can also be expressed as spatial whitening of the received signal [13]. The selected $R$ branch outputs are maximum-ratio combined with orthogonal interference plus noise terms $n_{r}$. The resulting decision variable $U_{m}$ (see Fig. 1) is given by

$$
\begin{aligned}
U_{m} & =d_{m} \sum_{r=1}^{R} \sum_{l=0}^{L}\left|h_{r l}^{w}\right|^{2} S F E_{P c}+\sum_{r=1}^{R} n_{r} \\
& =d_{m} \operatorname{trace}\left(\mathbf{H R}_{w, s}^{-1} \mathbf{H}^{H}\right) S F E_{P c}+\sum_{r=1}^{R} n_{r}
\end{aligned}
$$

where $h_{r l}^{w}$ is the amplitude of the $l$ th tap of the channel computed at sensor $r$ (after whitening with $\mathbf{R}_{w, s}^{-1 / 2}$ ), $S F$ is the spreading factor (or processing gain) and $E_{p c}$ stands for the chip pulse energy. The trace(.) operator returns the sum of the elements in the diagonal of its argument. The variance, for binary constellation, is given by (since the noise is spatially whitened after the spatial processor)

$$
\sigma_{n}^{2}=\sum_{r=1}^{R} \sigma_{r}^{2}=\frac{E_{P c} S F}{2} \operatorname{trace}\left(\mathbf{H R}_{w, s}^{-1} \mathbf{H}^{H}\right)
$$

and the BER yields ( $R$ is the number of branches in Fig. 1)

$$
\begin{aligned}
\operatorname{BER}_{\mathrm{NIMI}} & =Q\left(\sqrt{2 E_{b} \operatorname{trace}\left(\mathbf{H R}_{w, s}^{-1} \mathbf{H}^{H}\right)}\right) \\
& =Q\left(\sqrt{2 E_{b} \operatorname{trace}\left(\sum_{i=1}^{R} \lambda_{i} \mathbf{v}_{i} \mathbf{v}_{i}^{H}\right)}\right) \\
& =Q\left(\sqrt{2 E_{b} \sum_{i=1}^{R} \lambda_{i}}\right)
\end{aligned}
$$

where $E_{b}$ is the energy per bit and $\lambda_{i}$ are the $R$ nonzero eigenvalues of the Hermitian matrix $\mathbf{H R}_{w, s}^{-1} \mathbf{H}^{H}$.

\section{B. EMDIR Receiver}

For the $M$ sensor EMDIR, one has to analyze the number $R$ of significant branches (the number of different nonzero eigenvalues). The $R$ outputs are combined with uncorrelated interference plus noise terms, as it has been state in Property 1 . If the resulting decision variable is considered Gaussian distributed

$$
\begin{aligned}
U_{m} & =d_{m} \sum_{i=1}^{R} \tilde{\mathbf{h}}_{i}^{H} \tilde{\mathbf{h}}_{i} S F E_{P c}+\sum_{i=1}^{R} n_{i} \\
& =\left\{\tilde{\mathbf{h}}_{i}^{H} \tilde{\mathbf{h}}_{i}=1\right\}=d_{m} R S F E_{P c}+\sum_{i=1}^{R} n_{i}
\end{aligned}
$$

where $\tilde{\mathbf{h}}_{i}=\mathbf{H b}_{i}$. Since the noises at each branch are uncorrelated (see Property 1) and assumed all temporally white, the variance is now computed as

$$
\sigma_{n}^{2}=\sum_{i=1}^{R} \sigma_{i}^{2}=\frac{S F E_{P c}}{2} \sum_{i=1}^{R} \lambda_{i}
$$

where $\lambda_{j}$ is the noise power associated to the $j$ th branch

$$
\begin{aligned}
\mathbf{R}_{w, s} \mathbf{b}_{j} & =\lambda_{j} \frac{1}{N} \mathbf{H}^{H} \mathbf{D}^{H} \mathbf{D H} \mathbf{b}_{j} \Rightarrow \frac{1}{\lambda_{j}} \mathbf{b}_{j}=\mathbf{R}_{w, s}^{-1} \mathbf{H}^{H} \mathbf{H} \mathbf{b}_{j} \\
\frac{1}{\lambda_{j}} \tilde{\mathbf{h}}_{j} & =\mathbf{H R}_{w, s}^{-1} \mathbf{H}^{H} \tilde{\mathbf{h}}_{j} \Rightarrow \tilde{\mathbf{H}} \Lambda^{-1}=\mathbf{H R}_{w, s}^{-1} \mathbf{H}^{H} \tilde{\mathbf{H}} .
\end{aligned}
$$

Using Property $2\left(\tilde{\mathbf{H}} \tilde{\mathbf{H}}^{H}=\mathbf{I}\right)$, then it turns out that

$$
\begin{aligned}
\sigma_{n}^{2} & =\sum_{i=1}^{R} \sigma_{i}^{2}=\frac{S F E_{P c}}{2} \operatorname{trace}\left(\mathbf{H R}_{w, s}^{-1} \mathbf{H}^{H}\right)^{\#} \\
& =\frac{S F E_{P c}}{2} \sum_{i=1}^{R} \lambda_{i}
\end{aligned}
$$

where (.)\# is the pseudoinverse operator. Note that the $\lambda$ are the same as in (21). The BER yields

$$
\begin{aligned}
\mathrm{BER}_{\mathrm{EMDIR}} & =Q\left(\sqrt{\frac{2 E_{b} R^{2}}{\operatorname{trace}\left(\mathbf{H R}_{w, s}^{-1} \mathbf{H}^{H}\right)^{\#}}}\right) \\
& =Q\left(\sqrt{\frac{2 E_{b} R^{2}}{\operatorname{trace}\left(\sum_{i=1}^{R} \frac{1}{\lambda_{i}} \mathbf{v}_{i} \mathbf{v}_{i}^{H}\right)}}\right) \\
& =Q\left(\sqrt{\frac{2 E_{b} R^{2}}{\sum_{i=1}^{R} \frac{1}{\lambda_{i}}}}\right) .
\end{aligned}
$$

Using the fact that the eigenvalues are positive and that, in this case, the arithmetic mean is always larger than the harmonic mean, we conclude that (for the Gaussian noise case) $\mathrm{BER}_{\mathrm{EMDIR}} \geq \mathrm{BER}_{\mathrm{NIMI}}$, where equality holds if $R=1$. In this case, the receivers are the same.

\section{VRAKE Receiver}

For the sake of completeness let us derive the BER for the $M$ sensor VRAKE receiver. The $M$ branch outputs are combined 
with orthogonal interference plus noise terms. If the resultant decision variable can also be considered Gaussian distributed

$$
\begin{aligned}
U_{m} & =d_{m} \sum_{r=1}^{M} \sum_{l=0}^{L}\left|h_{r l}\right|^{2} S F E_{P c}+\sum_{r=1}^{M} n_{r} \\
& =d_{m} \operatorname{trace}\left(\mathbf{H}^{H} \mathbf{H}\right) S F E_{P c}+\sum_{r=1}^{M} n_{r} .
\end{aligned}
$$

If the noise is spatially and temporally uncorrelated, its variance is now computed as

$$
\sigma_{n}^{2}=\sum_{r=1}^{M} \sigma_{r}^{2}=\frac{E_{P c} S F N_{0}}{2} \operatorname{trace}\left(\mathbf{H}^{H} \mathbf{H}\right)
$$

and the BER yields

$$
\mathrm{BER}_{\text {VRAKE, white noise }}=Q\left(\sqrt{\frac{2 E_{b}}{N_{0}} \operatorname{trace}\left(\mathbf{H}^{H} \mathbf{H}\right)}\right) .
$$

If the noise is not white, then (28) is not valid. The true noise power is then given by

$$
\begin{aligned}
\sigma_{n}^{2} & =\frac{E_{b}}{2} \mathbf{1}^{T} E\left\{\mathbf{n n}^{H}\right\} \mathbf{1} \\
& =\frac{E_{b}}{2} \mathbf{1}^{T} \tilde{\mathbf{H}}^{H}\left(\mathbf{R}_{w, s} \otimes \mathbf{R}_{w, t}\right) \tilde{\mathbf{H}} \mathbf{1} \\
& =\frac{E_{b}}{2} \operatorname{vec}(\mathbf{H})^{H}\left(\mathbf{R}_{w, s} \otimes \mathbf{R}_{w, t}\right) \operatorname{vec}(\mathbf{H})
\end{aligned}
$$

where vector $\mathbf{n}$ collects the noise at each sensor output, $\mathbf{R}_{w, s}$ is the spatial covariance matrix of the noise-plus-interference, $\mathbf{1}^{T}=[11 \cdots 1]$ and

$$
\tilde{\mathbf{H}}^{H}=\left[\begin{array}{ccc}
\mathbf{h}_{0}^{H} & & \mathbf{0} \\
& \ddots & \\
\mathbf{0} & & \mathbf{h}_{M}^{H}
\end{array}\right] .
$$

If the noise is temporally white the equation can be further reduced to

$$
\begin{aligned}
\sigma_{n}^{2} & =\frac{E_{b}}{2} \mathbf{1}^{T} E\left(\mathbf{n n}^{T}\right) \mathbf{1} \\
& =\frac{E_{b}}{2} \mathbf{1}^{T} \tilde{\mathbf{H}}^{H}\left(\mathbf{R}_{w, s} \otimes \mathbf{I}\right) \tilde{\mathbf{H}} \mathbf{1} \\
& =\frac{E_{b}}{2} \operatorname{vec}(\mathbf{H})^{H}\left(\mathbf{R}_{w, s} \otimes \mathbf{I}\right) \operatorname{vec}(\mathbf{H}) \\
& =\frac{E_{b}}{2} \operatorname{trace}\left(\mathbf{H R}_{w, s} \mathbf{H}^{H}\right) .
\end{aligned}
$$

Finally, the BER is given by

$$
\mathrm{BER}_{\mathrm{VRAKE}}=Q\left(\sqrt{2 E_{b} \frac{\operatorname{trace}^{2}\left(\mathbf{H}^{H} \mathbf{H}\right)}{\operatorname{trace}\left(\mathbf{H R}_{w, s} \mathbf{H}^{H}\right)}}\right) .
$$

After some manipulations it is possible to check that $\mathrm{BER}_{\mathrm{VRAKE}}$ is always greater than or equal (with equality when noise is spatially white) to $\mathrm{BER}_{\mathrm{NIMI}}$ when $R=M$ (see Appendix II).

\section{Experimental Performance Evaluation}

\section{A. Propagation Channel Model}

In order to evaluate the receiver in a realistic mobile scenario, we have carried out simulations based on a Gaussian sta- tionary uncorrelated hypothesis for the channel, assuming independence between angular and Doppler spread, as it has been experienced from measurements taken in downtown Stockholm in the 1.8-GHz band [9]. There, it is empirically shown that azimuth spectrum follows a Laplacian law, along with Gaussian distribution for the directions of arrival $(\phi)$ around the mean angular position of the user. The angular spread (that is the standard deviation of the Gaussian, $\sigma_{\phi}$ ) is taken $8^{\circ}$. The number of rays impinging the array is fitted as a Poisson random variable of mean 25. The power delay spread will be based on the pedestrian and vehicular models for temporal spreading recommended in the SMG2 documents for UTRA [4]. The amplitude associated with each propagation path is a complex Gaussian random variable whose power decreases as the time delay and the angular direction of arrival with respect to the mobile position increase. A classical Clarke's bath-shaped Doppler spectrum is obtained by assuming multiple reflections close around the mobile. The carrier frequency is $2.0 \mathrm{GHz}$. All sensors have flat spatial response in a sectored area of $120^{\circ}$, and are linearly and uniformly spaced at $d / \lambda=0.5$.

\section{B. Simulations}

A set of simulations has been performed using up to nine users of spreading factor 16 in the uplink of the FDD mode of UTRA [4]. The uplink physical channels of the FDD mode of UTRA are as follows: each user generates at least one dedicated physical data channel (DPDCH) along with a single dedicated physical control channel (DPCCH). Each channel is spread with a different orthogonal variable spreading factor (OVSF) code at a chip rate of $4.096 \mathrm{Mchip} / \mathrm{s}$ and then in-phase and quadrature multiplexed in a quadrature phase-shift keying (QPSK) modulation. The existence of OVSF codes of different length allows the presence of different bit-rate users in the same cell. These complex symbols are scrambled by a mobile-station specific scrambling code using either codes from the very large Kasami set (to allow multiuser detection) or the Gold sequence of length 40960 (for single-user detection). Fig. 4 illustrates the signal generation scheme [4].

The UTRA air interface is designed to achieve a full $1: 1$ reuse, which implies no loss of capacity due to frequency planning. Since all users access the channel asynchronously, intercell and intracell users separation relies on the good correlation properties of the scrambling codes. Under this premises, all channelization OVSF codes are available to each user so many DPDCH can be set up to obtain the desired bit-rate (as shown in Fig. 4) with different weighting factors (noted with $\beta$ ). Due to the severity of the mobile channel, the dynamic range of received powers in the base station (BS) can be as high as $90 \mathrm{~dB}$. In order to avoid loss of efficiency due to the near-far problem, a tight control of the transmission power is introduced: every $0.625 \mathrm{~ms}$, the BS sends power control information through the forward link, and the mobiles update their transmitted power at the same frequency.

The frame structure is shown in Fig. 5. Each frame of duration $10 \mathrm{~ms}$ is split into 16 slots of duration $0.625 \mathrm{~ms}$, which corresponds to one power-control period. The DPDCH is used to transport data symbols, while the DPCCH is divided in three fields which are used, respectively, for channel estimation 
(PILOT), transmission of power control information (TPC) for the downlink and transport-format indicators (TFI). TPC and TFI occupy only the last $10 \%$ of the slot duration, so the receiver is able to estimate and track the channel for the first $90 \%$. Usually, the channel estimation is made using the pilot chips, and different amplitudes can be assigned to the DPDCH and the DPCCH channels.

The channel has been estimated using all the chips of the pilot, so the autointerference of the traffic is very low. All users are assumed to have controlled transmitted power with no errors. An error of $1 \mathrm{~dB}$ showed no difference in performance. $E_{b} / N_{o}$ is $15 \mathrm{~dB}$. The speeds of users are $3 \mathrm{~km} / \mathrm{h}$, for the pedestrian A and B channels and $50 \mathrm{Km} / \mathrm{h}$ for the vehicular channel. The EMDIR, with different number of eigenvectors, the conventional VRAKE and NIMI (with $R=M$ ) have been tested and its performance plotted in Fig. 2.

In order to evaluate the alternative between multiuser and single user detection in the UL of the FDD mode, a comparison of the different proposed receivers with the multiuser receiver zero-forcing is done (the scrambling codes used are the Kasami sequences in the later case).

\section{Evaluation of Results}

In all cases, the performance was superior to the conventional VRAKE receiver, so we can conclude that substantial gain from the use of spatial beamforming is achieved. EMDIR also performs better than the NIMI receiver in all cases. Not surprisingly, a significant reduction in BER is obtained for the EMDIR when using two beamformers, in particular for the pedestrian B and the vehicular channel, whose delay spread is larger than for the pedestrian A [4].

This is verified in Fig. 3, where the cumulative function of the ratio between the increasing eigenvalues and the maximum eigenvalue of the MDIR receiver is depicted, for different number of active users. Clearly, the second eigenvalue (that is, the SINR associated to the output of the second beamformer) is always significant, although it decreases slightly as the number of active users increase. The third eigenvalue seems to be only significant for a low number of users, so it may be discarded.

On the other hand, performance is somewhat reduced when the number of beamformers is larger (four beamformers), in the results obtained for the pedestrian channel. The reduction in performance agrees with a known result from estimation theory, that is, a rank reduction provides improved efficiency.

In other words, more delay and azimuth dispersive channels allow further diversity gains, so modulations with larger bandwidth (higher chip rate) benefit more of temporal dispersion for the same dispersive channel.

Finally, in the situation described, the EMDIR exhibits quite similar performance to the zero-forcing multiuser detector.

\section{CONCLUSION}

An improved version of the MDIR receiver has been presented and compared in terms of complexity and performance with the NIMI. It can be concluded in the following.

- Although the structure of the receiver is the same (multiple beamformers and coherent combining of their
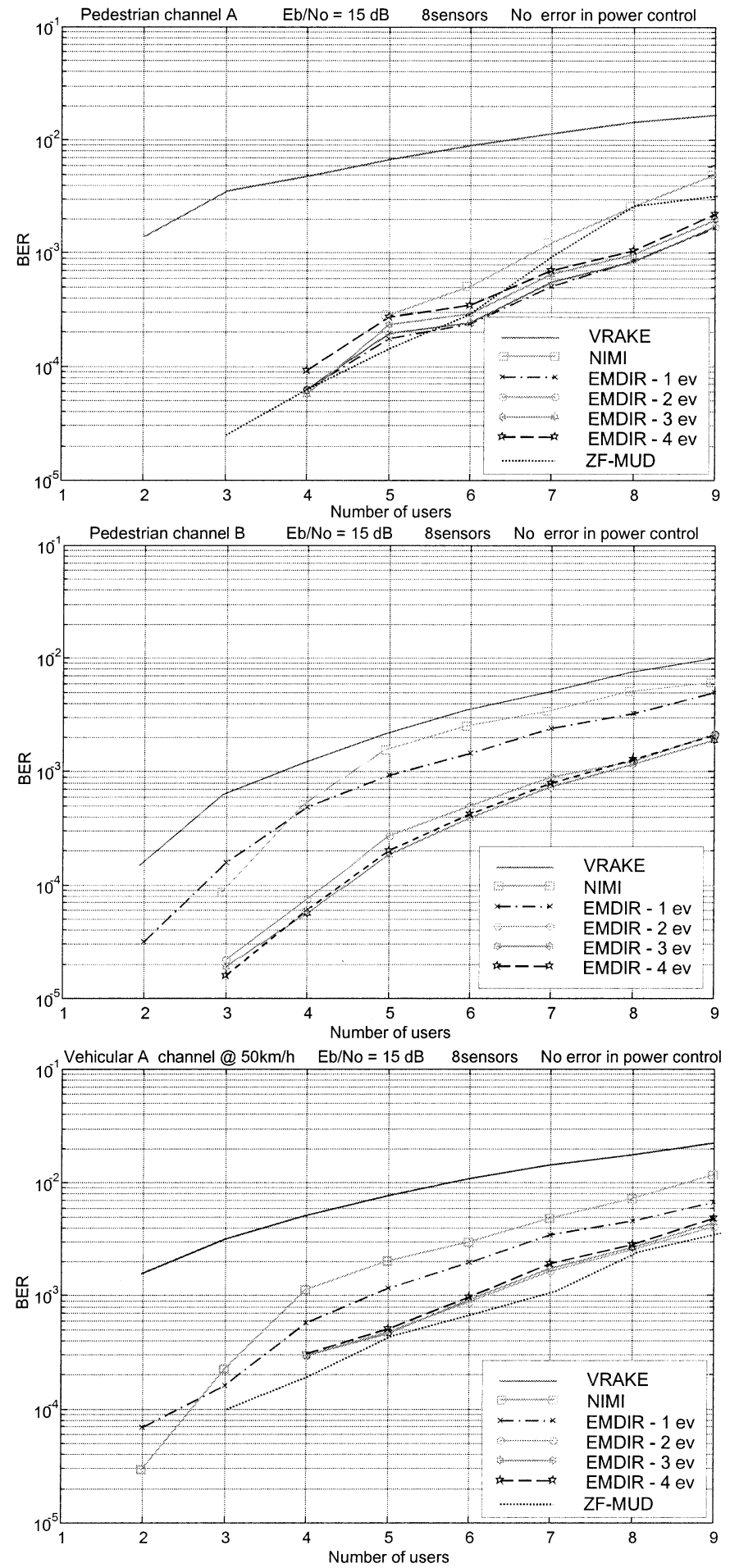

Fig. 2. Probability of error for a different number of active users, all transmitting controlled power, for the different receivers. Pedestrian channels (top-middle) and vehicular channel (bottom).

outputs) the EMDIR and W-VRAKE are not equivalent, due to the different spatial combiners $\mathbf{b}$. In two cases, both receivers achieve the same BER for Gaussian scenario: when $\mathbf{R}_{w}$ is diagonal and, when $R=1$ is chosen. Otherwise, according to (21) and (26), NIMI outperforms EMDIR, which is not an unexpected result since we have derived the equations considering Gaussian noise-plus-interference. 

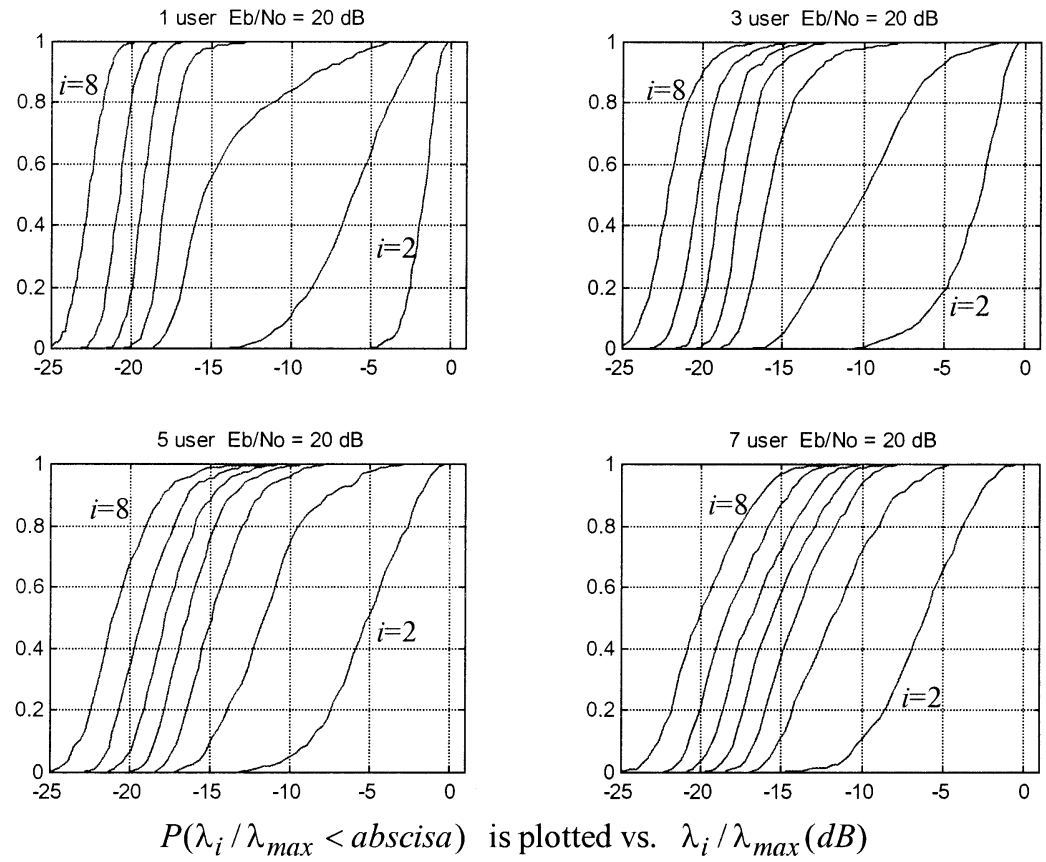

Fig. 3. Cumulative functions of the ratio of the MDIR eigenvalues to the maximum eigenvalue, for the vehicular channel.

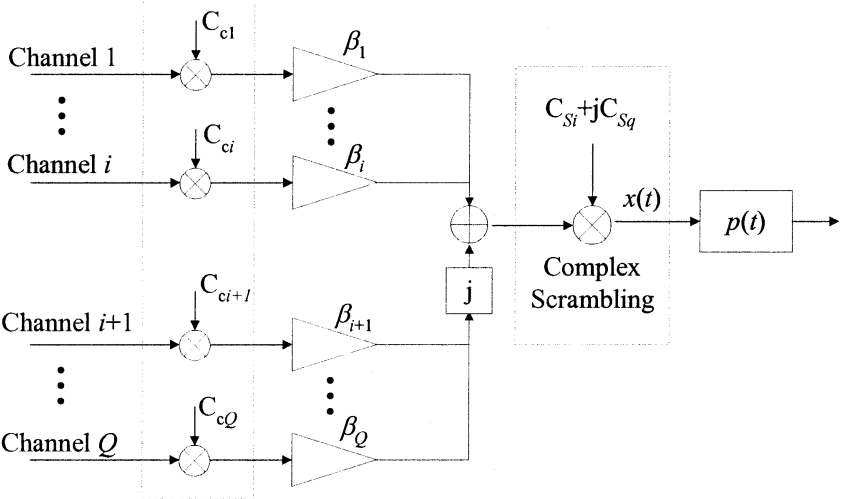

Channelization

Fig. 4. Modulated signal generation.

- For the non-Gaussian case, simulations indicate that EMDIR outperforms NIMI. Its performance depends on the noise-plus-interference spatial distribution as well as the desired signal spatial-temporal distribution: highly dispersive channels yield a matrix $\mathbf{H}^{H} \mathbf{H}$ with similar eigenvalues, so the use of multiple branches becomes interesting. In this case, (26) might eventually be used to have an estimate of the gain obtained when using an additional diversity branch.

- The NIMI and weighted VRAKE receivers requires the computation of the noise-plus-interference matrix, which is numerically difficult to compute (see Appendix I). On the contrary, the EMDIR receiver may be easily reformulated using $\mathbf{R}_{y}$ instead of $\mathbf{R}_{w}$ [6], so no positive matrix differences have to be computed.

- The EMDIR with the proper choice of the order performs very much like the zero-forcing multiuser detector.
APPENDIX I

ESTIMATION OF THE NOISE-PLUS-INTERFERENCE MATRIX

The estimation of the noise-plus-interference matrix is not a straightforward problem for the uplink in the FDD mode of UTRA, since we must ensure that 1) the computed matrix is definite semipositive and 2) there are no traces of the data channel in the computed matrix. We propose here a regularization device which will allow a proper estimation of $\mathbf{R}_{w}$ and a fair comparison of the receivers. Let us use the model in s (10) and (11). Note however, that according to the signal structure of the FDD mode of UMTS, we cannot completely determine matrix $\mathbf{D}$ beforehand since it contains the known chips of the pilot channel $\mathbf{D}_{p}$, but also the unknown chips of the traffic channel $\mathbf{D}_{t}$

$$
\mathbf{D}=\beta_{1} \mathbf{D}_{p}+\beta_{2} \mathbf{D}_{t}
$$

where $\beta_{1}$ is the weighting factor associated to the pilot (known) chips and $\beta_{2}$ is the one associated to the traffic (unknown) chips. First of all, it is worth mentioning that the channel in (9) may be estimated consistently by applying a least-square estimation as $\hat{\mathbf{H}}=\beta_{1}^{-1}\left(\mathbf{D}_{p}^{H} \mathbf{D}_{p}\right)^{-1} \mathbf{D}_{p}^{H} \mathbf{Y} \cong\left(2 \beta_{1}\right)^{-1}(N-L+1)^{-1} \mathbf{D}_{p}^{H} \mathbf{Y}$ in which uncorrelation between known and unknown chips is assumed. Under these premises, the space correlation matrix of interference and noise can be computed ergodically as

$$
\begin{aligned}
\hat{\mathbf{R}}_{w, s} & =\mathbf{Y}^{H} \mathbf{Y}-\mu \mathbf{H}^{H} \mathbf{D}^{H} \mathbf{D H} \\
& \cong \mathbf{Y}^{H} \mathbf{Y}-2 \mu\left(\beta_{1}^{2}+\beta_{2}^{2}\right)(N-L+1) \mathbf{H}^{H} \mathbf{H} \\
& =\hat{\mathbf{R}}_{y, s}-\mu \hat{\mathbf{R}}_{s, s}
\end{aligned}
$$

where uncorrelation in the complex symbols and also between the traffic and pilot channels has been assumed, and taken into account the different amplitudes of the pilot and traffic channels. The term $\mu$ is included with the following purpose: one of the shortcomings of the matrix substraction is that it may lead to 


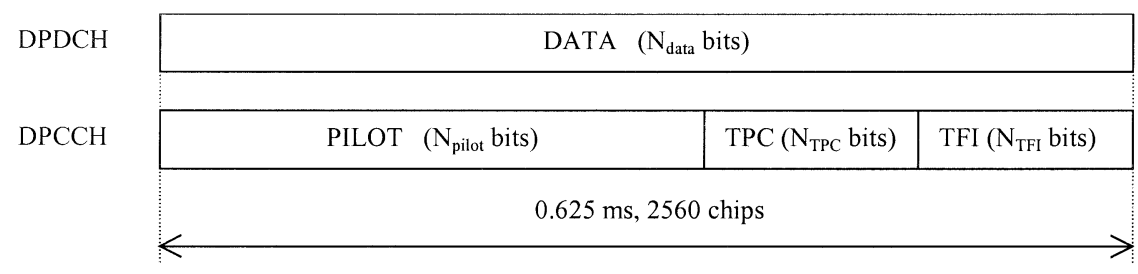

Fig. 5. Slot structure for the uplink in the FDD mode.

nonpositiveness due to estimation errors. Then, the $\mu$ factor can be chosen conveniently

$$
\mathbf{z}^{H} \hat{\mathbf{R}}_{w, s} \mathbf{z}=\mathbf{z}^{H} \hat{\mathbf{R}}_{y, s} \mathbf{z}-\mu \mathbf{z}^{H} \hat{\mathbf{R}}_{s, s} \mathbf{z}>0 \quad \forall \mathbf{z} .
$$

If this equation has to be positive definite for every possible vector $\mathbf{z}$, then the value of $\mu$ has to be smaller than the minimum value of the Rayleigh quotient, that is, smaller than the minimum eigenvalue of the matrix pencil

$$
\mu<\min \left\{\frac{\mathbf{z}^{H} \hat{\mathbf{R}}_{y, s} \mathbf{z}}{\mathbf{z}^{H} \hat{\mathbf{R}}_{s, s} \mathbf{z}}\right\} .
$$

\section{APPENDIX II}

\section{COMPARISON OF BER IN VRAKE AND NIMI RECEIVERS}

It is also possible to compare the NIMI and the VRAKE in the following way (assuming that all the eigenvalues of $\mathbf{R}_{w, s}^{-1}$ are non-null):

$\operatorname{trace}\left(\mathbf{H}^{H} \mathbf{H} \mathbf{R}_{w, s}\right)=\operatorname{trace}\left(\mathbf{B}^{H} \mathbf{A} \Lambda \mathbf{A}^{H} \mathbf{B} \Sigma\right)=\sum_{i} a_{i} \alpha_{i}$

$\operatorname{trace}\left(\mathbf{H}^{H} \mathbf{H} \mathbf{R}_{w, s}^{-1}\right)=\operatorname{trace}\left(\mathbf{B}^{H} \mathbf{A} \Lambda \mathbf{A}^{H} \mathbf{B} \Sigma^{-1}\right)=\sum_{i} a_{i} / \alpha_{i}$

where $\mathbf{H}^{H} \mathbf{H}=\mathbf{A} \Lambda \mathbf{A}^{H}, \mathbf{R}_{w, s}=\mathbf{B} \Sigma \mathbf{B}^{H}$, and $a_{i}$ are the elements in the trace of the Hermitian matrix $\mathbf{B}^{H} \mathbf{A} \Lambda \mathbf{A}^{H} \mathbf{B}$. The circular product property of the trace is used in the first equality. Due to definite positiveness of matrix involved, all $a$ and $\alpha$ are positive magnitudes. Therefore, the following comparison is in order [as taken from (21) and (32)]:

$$
\begin{gathered}
\operatorname{trace}\left(\mathbf{H}^{H} \mathbf{H} \mathbf{R}_{w, s}^{-1}\right) \triangleright \triangleleft \frac{\operatorname{trace}\left(\mathbf{H}^{H} \mathbf{H}\right)^{2}}{\operatorname{trace}\left(\mathbf{H}^{H} \mathbf{H} \mathbf{R}_{w, s}\right)} \\
\sum_{i} a_{i} / \alpha_{i} \triangleright \triangleleft \frac{\left(\sum_{i} a_{i}\right)^{2}}{\sum_{i} a_{i} \alpha_{i}} \\
\sum_{i \neq j} a_{i} a_{j}\left(\frac{\alpha_{i}}{\alpha_{j}}+\frac{\alpha_{j}}{\alpha_{i}}-2\right) \triangleright \triangleleft 0
\end{gathered}
$$

Hence, since all terms $a_{i}$ are positive, the term in brackets is positive and the whole is greater than zero. Therefore, $\mathrm{BER}_{\text {VRAKE }} \geq \mathrm{BER}_{\mathrm{NIMI}}$.

\section{ACKNOWLEDGMENT}

The authors would like to thank D. Pérez-Palomar for the careful reading of the manuscript.

\section{REFERENCES}

[1] P. Balaban and J. Salz, "Optimum diversity combining and equalization in digital data transmission with applications to cellular mobile radio? Part I: Theoretical considerations," IEEE Trans. Commun., vol. 40, pp. 885-907, May 1992.

[2] J. W. Brewer, "Kronecker products and matrix calculus in system theory," IEEE Trans. Circuits Syst., vol. 25, pp. 772-781, Sept. 1978.

[3] G. H. Golub and C. F. Van Loan, Matrix Computations. Baltimore, MD: The John Hopkins Univ. Press, 1983.

[4] H. Holma and A. Toskala, Eds., WCDMA for UMTS. Radio Access for Third Generation Mobile Communications. New York: Wiley, 2000.

[5] P. Jung and J. Blanz, "Joint detection with coherent receiver antenna diversity in CDMA mobile radio systems," IEEE Trans. Veh. Technol., vol. 44, pp. 76-88, Feb. 1995.

[6] M. A. Lagunas, J. Vidal, and A. I. Perez, "Joint array combining and MLSE for single-user receivers in multipath Gaussian multiuser channels," IEEE J. Select. Areas Commun., vol. 18, pp. 2252-2259, Nov. 2000

[7] X. Mestre and J. R. Fonollosa, "Algorithms for flexible multistandard array processing: Part 3," Sunbeam, Deliverable D711, AC347/UPC/A72/PI/I007/b1, ACTS 0347 SUNBEAM.

[8] J. Paulraj and C. B. Papadias, "Space-time processing for wireless communications," IEEE Signal Processing Mag., pp. 49-83, Nov. 1997.

[9] K. Pedersen, P. Mogensen, and B. Fleury, "A stochastic model of the temporal and azimuthal dispersion seen at the base station in outdoor propagation environment," IEEE Trans. Veh. Technol., vol. 49, pp. 437-447, Mar. 2000.

[10] M. Stojanovic and Z. Zvonar, "Multichannel processing of broad-band multiuser communication signals in shallow water acoustic channels," IEEE J. Oceanic Eng., vol. 21, no. 2, pp. 156-166, Apr. 1996.

[11] W. Van Etten, "Maximum-likelihood receiver for multiple channel transmission systems," IEEE Trans. Commun., pp. 276-283, Feb. 1976.

[12] S. Verdú, Multiuser Detection. Englewood Cliffs, NJ: Prentice-Hall, 1998.

[13] J. Vidal, M. Cabrera, and A. Agustin, "Full exploitation of diversity in space-time MMSE receivers," in Proc. VTC-2000, Boston, MA, Sept. 2000, pp. 2497-2502.

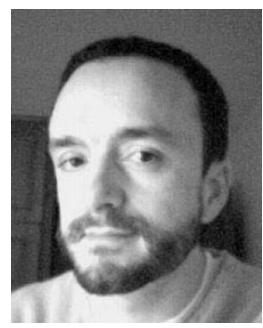

Josep Vidal (S'88-M'91-A'95-M'02) received the telecommunication engineering and Ph.D. degrees from the Polytechnic University of Catalonia (UPC), Barcelona, Spain, in 1989 and 1993, respectively.

In 1988, he was with Cognivision Research, S.L., Barcelona, Spain. From 1989 to 1990, he was with the LSI, Ecole Polytechnique de Lausanne, Switzerland, as Research Assistant. In 1991, he was the recipient of a Ministry of Education grant to complete the Ph.D. dissertation and joined the Signal Theory and Communications Department, University of Catalonia. In 1993, he became a Lecturer and since 1996, he has been an Associate Professor at University of Catalonia. His current research interest is in signal processing applied to different aspects of mobile communication systems. He has published over 60 papers on these areas in international journals and conferences. He has been leading the University of Catalonia participation in project SATURN and is Coordinator of project ROMANTIK, both belonging to the 5th framework program of the European Commission (EC). He has been co-organizer of the IEEE Signal Processing/ATHOS Workshop on Higher Order Statistics in Begur, Spain (1995), and the European Commission-sponsored IST Mobile Communications Summit in Sitges, Spain (2001). 


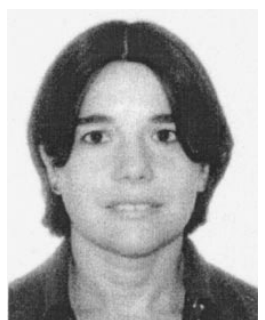

Margarita Cabrera received the electrical engineering degree and the Ph.D. degree from the Polytechnic University of Catalonia (UPC), Barcelona, Spain, in 1986 and 1991, respectively.

Since 1992, she has been an Associate Professor at the UPC, where she teaches undergraduate courses in analog and digital communications and graduate courses in signal processing. Her current research interests are in general signal processing applications and include mobile communication systems, code division multiple access (CDMA), and synchronization in digital communications. Recently, she has participated in several research projects supported by the European Commission (EC) in the areas of UMTS and GSM.

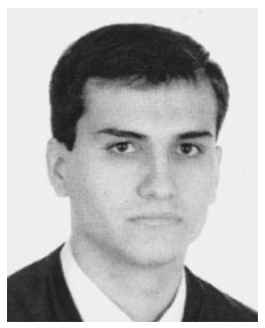

Adrían Agustín de Dios received the telecommunication engineering degree in 2000 and is currently working toward the electronics engineering degree from the Universitat Politècnica de Catalunya, Spain.

Since 2000, he has been with Indra-Espacio, Barcelona, Spain, where he has been engaged in the research and development of code synchronization techniques for DS-CDMA.

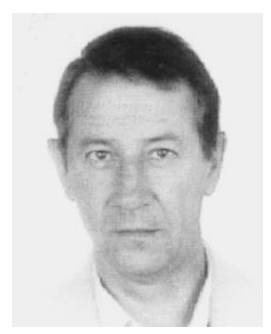

Miguel Angel Lagunas (S'73-M'78-SM'89-F'97) was born in Madrid in 1951. He received the telecommunications engineer degree in 1973 from Universidad Politécnica de Madrid (UPM), Madrid, Spain and the Ph.D. degree in telecommunications from Universidad Politécnica de Barcelona (UPB), Barcelona, Spain.

From 1971 to 1973, he was a Research Assistant at the Semiconductor Lab. (ETSIT), Madrid, Spain. From 1973 to 1979, he was a Teaching Assistant in Network Synthesis and Semiconductor Electronics, an Associate Professor on digital signal processing from 1979 to 1982, and since 1983 a Professor teaching courses in signal processing, array processing, and digital communications. He was Project Leader of high-speed SCMA (1987-1989) and ATM cable network (1994-1995), Co-Director of the first projects for the European Spatial Agency and the European Union providing engineering demonstration models on smart antennas for satellite communications using DS and FH systems (1986) and mobile communications GSM (Tsunami, 1994). From 1986 to 1989, he was Vice-President of Research, Universitat Politècnica de Catalunya (UPC), Spain, Vice-Secretary General of Research (CICYT-Spain) from 1995 to 1996. His research activity is devoted to spectral estimation, adaptive systems, and array processing. His technical activities are in advanced front-ends for digital communications combining spatial with frequency-time and coding diversity.

Dr. Lagunas was Member at large of Eurasip (1990) and Elected Member of the Academy of Engineers of Spain (1998). He received a Fullbright Postdoctoral grant from the University of Boulder, Boulder, CO, in 1981. 